\title{
Delivering WHO's Life-Saving Information in Real-Time During a Pandemic Through an Online Learning Platform: Evidence from Global Use
}

\author{
Heini UTUNEN ${ }^{\mathrm{a}, 1}$, Richelle GEORGE ${ }^{\mathrm{a}}$, Ngouille NDIAYE ${ }^{\mathrm{a}}$, Anna TOKAR ${ }^{\mathrm{a}}$, Melissa \\ ATTIAS $^{\mathrm{a}}$ and Gaya GAMHEWAGE ${ }^{\mathrm{a}}$ \\ ${ }^{\text {a } W o r l d ~ H e a l t h ~ O r g a n i z a t i o n}$
}

\begin{abstract}
Accessibility, multilingualism and real-time knowledge transfer are the tenets of the OpenWHO learning platform. A descriptive analysis was conducted using anonymised statistical datasets retrieved from the OpenWHO reporting system to understand global use trends. When examining OpenWHO use in the areas with the highest burden of COVID-19 cases, a strong correlation was identified between the burden of COVID-19 and OpenWHO user activity. Further, health professionals are the top users of COVID-19 materials. The massive demand for OpenWHO courses confirms the need for real-time learning materials in accessible, multilingual formats during a pandemic. Learning is a global public good and is an essential component of the response to health emergencies.
\end{abstract}

Keywords. COVID-19, e-learning, online learning, pandemic, World Health Organization

\section{Introduction}

For the World Health Organization (WHO), a pandemic was considered a question of when rather than if. Pandemic preparedness was systematic and included the Pandemic Influenza Preparedness (PIP) Framework, which supported readiness work in-countries, and public-private partnerships. The 2014-2016 Ebola outbreak in West Africa made it clear that the world needed a learning platform tailored to disseminating knowledge to respond to health emergencies.

In 2016, WHO's Health Emergencies Learning and Capacity Development (LCD) team began working on launching an online learning platform to facilitate the delivery of life-saving knowledge to the frontline. This was done in anticipation of a worldwide pandemic, during which WHO would need to rapidly scale up global dissemination of reliable, impactful technical guidance-based learning resources to health workers and affected communities. The PIP Framework and emergency funds supported this work to prepare for what was intended to be the world's widest-reaching online training response for use during health emergencies.

\footnotetext{
${ }^{1}$ Heini Utunen, Corresponding author, WHO Health Emergencies Programme, World Health Organization, Geneva, Switzerland; E-mail: utunenh@who.int.
} 
The LCD team collaborated with the Hasso Plattner Institute to create a learning platform enhanced by technology, the requirements for which were:

- The infrastructure necessary to scale up to withstand millions of learners

- User-friendly course design functions, making it easy for WHO technical experts to publish learning resources and guidance online in real-time

- Equitable access at the front line of health emergencies. Taking into account language needs, cultural preferences for learning, compromised connectivity, cost and other aspects of the digital divide, courses had to be available in lowbandwidth friendly formats, with the option to download materials for offline access, and offered free of charge.

In March 2017, OpenWHO.org was launched, with a pilot course on managing infectious hazards offered to test users from the WHO African Region. By December 2019 , there were 160,000 course registrations on the platform. The platform's self-paced courses, accessible from anywhere in the world, were helping to advance WHO's aim of strengthening staff competencies within the Organization, in ministries of health, emergency operations centres, and at the front line, enabling health workers to operate ethically, effectively and safely during health emergencies.

2020 brought with it the first pandemic in OpenWHO's lifetime. The LCD team took note of the early signs of the virus in January and prepared accordingly. In collaboration with WHO's coronavirus technical lead, the team transformed existing technical knowledge into an online, learning-friendly format. On 26 January, the first COVID-19 course was published on OpenWHO. In the nine months that followed, the pandemic put the platform to the test.

The COVID-19 pandemic caused medical schools and training centres worldwide to close, highlighting the urgent need for alternative approaches and channels of education, not only for health professionals but also for the general population. In line with all digital services, online learning has found new ground during the pandemic [14]. Research suggests that digital learning approaches can improve the knowledge of public health professionals [1-4]. These approaches are more effective at improving knowledge than no intervention [1-3], or at least as effective as traditional learning [3]. However, it has also been said that e-learning may make little or no difference in patient outcomes or health professionals' behaviours, skills or knowledge [4].

This study aims to examine the trends in OpenWHO use between June and September 2020 .

\section{Methods}

Data were obtained using anonymised statistical datasets retrieved from the OpenWHO reporting system. A descriptive analysis was conducted in Microsoft Excel to identify patterns in user characteristics and assess frequencies in terms of location, gender, language, age and profession, all of which are disclosed by the learner during the platform's registration process.

The paper focuses on the period between June and September 2020 to examine the users who had most recently joined OpenWHO at the time of the study. User activity data were captured by Google Analytics, as of August 2020. This data was then merged with a statistical dataset from the August 2020 edition of the WHO Health Emergencies Programme (WHE) Situation Report, which tallied the number of COVID-19 cases in each country. 


\section{Results}

Between January and October 2020, course registrations on OpenWHO skyrocketed from 160,000 to 4.5 million. During this time, the platform added 19 new courses, each covering a topic related to COVID-19. These courses were translated across 41 languages, adding up to 136 language versions of the 19 courses on offer.

\subsection{Correlation between number of COVID-19 cases and OpenWHO use}

The majority of those enrolled on OpenWHO's COVID-19 courses are based in the WHO South-East Asia Region (36.38\%) and the Americas Region (23.40\%). Out of the 20 countries with the highest number of course enrolments, ten are located in these regions; this includes the country with the highest recorded OpenWHO activity: India. In fact, almost one in three COVID-19 course users is from India (31.94\%).

When comparing the global patterns of OpenWHO use with the spread of COVID19, a strong correlation can be identified between the burden of COVID-19, as measured by the number of cases, and the intensity of OpenWHO user activity within these regions (Table 1). The 20 countries with the highest number of COVID-19 cases accounted for $82.55 \%$ of the global caseload. Fourteen of these (highlighted in blue in Table 1) are also among the 20 countries with the highest OpenWHO activity levels.

Table 1. The 20 countries with the highest number of COVID-19 cases and OpenWHO activity as of August 2020

\begin{tabular}{|c|c|}
\hline $\begin{array}{c}\text { Top } 20 \text { countries COVID-19 confirmed } \\
\text { cases } \\
\text { (\% total global confirmed cases }) \\
\end{array}$ & $\begin{array}{c}\text { Top } 20 \text { countries OpenWHO web sessions }{ }^{\mathbf{a}} \\
(\% \text { global OpenWHO activity) }\end{array}$ \\
\hline 1. United States of America (23.56\%) & 1. India $(21.16 \%)$ \\
\hline 2. Brazil (15.31\%) & 2. United States of America (6.21\%) \\
\hline 3. India $(14.25 \%)$ & 3. Mexico $(5.63 \%)$ \\
\hline 4. Russian Federation (3.98\%) & 4. Ecuador $(4.37 \%)$ \\
\hline 5. Peru $(2.53 \%)$ & 5. Colombia $(3.45 \%)$ \\
\hline 6. South Africa $(2.50 \%)$ & 6. Nigeria $(3.32 \%)$ \\
\hline 7. Colombia $(2.38 \%)$ & 7. Saudi Arabia $(2.78 \%)$ \\
\hline 8. Mexico $(2.36 \%)$ & 8. Bangladesh $(2.71 \%)$ \\
\hline 9. Spain $(1.77 \%)$ & 9. United Kingdom $(2.62 \%)$ \\
\hline 10. Chile $(2.38 \%)$ & 10. Argentina $(2.48 \%)$ \\
\hline 11. Argentina (1.58\%) & 11. Pakistan $(2.28 \%)$ \\
\hline 12. Iran $(1.50 \%)$ & 12. Philippines $(2.14 \%)$ \\
\hline 13. United Kingdom (1.34\%) & 13. Chile $(1.94 \%)$ \\
\hline 14. Saudi Arabia (1.26\%) & 14. Egypt (1.89\%) \\
\hline 15. Bangladesh (1.24\%) & 15. Iraq $(1.73 \%)$ \\
\hline 16. Pakistan $(1.19 \%)$ & 16. Peru $(1.63 \%)$ \\
\hline 17. Turkey $(1.07 \%)$ & 17. Spain $(1.63 \%)$ \\
\hline 18. Italy $(1.07 \%)$ & 18. Brazil $(1.45 \%)$ \\
\hline 19. France $(1.03 \%)$ & 19. Canada (1.38\%) \\
\hline 20 . Germany $(0.93 \%)$ & 20. South Africa $(1.31 \%)$ \\
\hline
\end{tabular}

The data suggest a strong correlation between the burden of disease and OpenWHO activity in countries like the United States of America and India. However, Brazil, the second hardest-hit country in terms of COVID-19 caseload, ranks $18^{\text {th }}$ for OpenWHO activity. For example, it is preceded by Nigeria, which does not even rank among the 20 
countries with the highest number of COVID-19 cases. Similarly, for Russia, Iran and some European countries, the data suggest that the high disease burden does not translate into a heightened use of the platform.

\subsection{OpenWHO user professions}

Between June and September 2020, there were 357,606 enrolments on COVID-19 courses from users new to OpenWHO. Of these, $98.42 \%$ disclosed their professions.

Healthcare professionals are the largest group enrolled on COVID-19 courses and account for $29.33 \%$ of total enrolments. Together with users from health institutes, ministries and organisations, they make up two-fifths of the total learners.

\subsection{Multilingualism}

OpenWHO's leading core value is multilingualism. By the end of August 2020, COVID19 courses had been translated across 39 languages. English courses were the most popular, comprising $73.27 \%$ of all enrolments, followed by Spanish (14.84\%). French, Portuguese and Arabic accounted for between 1.50 and 2.50\% each, Russian $0.60 \%$ and Chinese $0.35 \%$. All other languages combined made up $5 \%$ of the total enrolments.

\section{Discussion}

Being "fast, first and frequent" - disseminating life-saving information in real-time - is critical during a pandemic such as this one. The demand for learning has been illustrated by the rapid and unprecedented increase in enrolments on OpenWHO's COVID-19 courses. The correlation between OpenWHO use and COVID-19 caseload demonstrates the demand for reliable, technical knowledge about COVID-19 in the areas most affected. It is interesting to note that the pre-pandemic MOOC penetration trends greatly benefit the efficient reach of the OpenWHO response, as the hardest-hit regions notably the United States and India, are the same areas with the highest OpenWHO activity [5]. The significant uptake of OpenWHO's online resources in Nigeria can be explained by the previous success of the Ebola education and e-training campaigns that exemplified the use of social media in the health field to dispel rumors and misinformation about the disease in 2014 [6]. The remarkable case of Nigeria suggests that past experience using eHealth/mHealth in past disease outbreaks might factor into its propensity to use online resources as an effective part of the response.

In terms of occupation, healthcare professionals are the largest learner group on OpenWHO, accounting for nearly one-third of users, illustrating the existing demand for e-learning among health care workers. Several studies have reported that the current COVID-19 crisis can be seen as a "black swan moment" concerning health professionals' training and the role of digital learning [7-9]. Digital learning allows participants to work at their own pace, and thus, it is more flexible, self-directive, and inclusive compared to traditional learning strategies. These approaches are an alternative avenue to educate the adult population without forcing them to choose between their professional and familial lives or requiring them to return to university to improve their knowledge and selfconfidence $[2,10-11]$. 
Through collaboration with translators based in WHO country and regional offices, the LCD team has increased accessibility to learning by offering COVID-19 courses across 41 languages. Given its life-saving potential, every effort must be made to increase equitable access to learning for those who need it most. As argued by Aldohyan, the focus of learning strategies should not be the content of materials, but their accessibility and structure [7].

This study's findings should be interpreted in light of its methodological limitations, including the descriptive nature of data collected and the absence of statistical tests performed. Caution should be taken if generalising findings to different contexts.

\section{Conclusions}

Learning and capacity development have rightfully become part and parcel of WHO's international response to health emergencies. The trends in OpenWHO use prove that knowledge and education are global public goods. Moreover, in combination with learning science, technology can be harnessed to create high-quality learning for all. During the disruption of a health emergency, the success of the public health response relies in part on the speed, accuracy, reach, and impact of the right information.

\section{References}

[1] Brown A, Barnes C, Byaruhanga J, McLaughlin M, et al. Effectiveness of Technology-Enabled Knowledge Translation Strategies in Improving the Use of Research in Public Health: Systematic Review. J Med Internet Res. 2020; 22(7): e17274.

[2] Martinengo L, Ying NYJ, Markandran KDO, et al. Digital health professions education on chronic wound management: a systematic review. International journal of nursing studies. 2019; 104:103512.

[3] Tudor Car L, Soong A, Kyaw BM, et al. Health professions digital education on clinical practice guidelines: a systematic review by Digital Health Education collaboration. BMC medicine. 2019; 17(1):139-16.

[4] Vaona A, Banzi R, Kwag KH, et al. E-learning for health professionals. Cochrane Database of Systematic Reviews. 2018; 1.

[5] Chauhan J. An Overview of MOOC in India. International Journal of Computer Trends and Technology. 2017;49(2):111.

[6] Carter M. How Twitter may have helped Nigeria contain Ebola. BMJ. 2014;349(nov19 3):g6946-g6946.

[7] Aldohyan M, Al-Rawashdeh N, Sakr FM, et al. The perceived effectiveness of MERS-CoV educational programs and knowledge transfer among primary healthcare workers: a cross-sectional survey. BMC Infectious Diseases. 2019; 19(273).

[8] Fernandez-Diaz E, Iglesias-Sanchez PP, Jambrino-Maldonado C. Exploring WHO Communication during the COVID 19 Pandemic through the WHO Website Based on W3C Guidelines: Accessible for All? International journal of environmental research and public health. 2020; 17(16):5663.

[9] Chick RC, Clifton GT, Peace KM, et al. Using Technology to Maintain the Education of Residents During the COVID-19 Pandemic. Journal of Surgical Education. 2020; 77(4):729-32.

[10] Regmi K, Jones L. A systematic review of the factors - enablers and barriers - affecting e-learning in health sciences education. BMC Med Educ. 2020; 20(1):91.

[11] Carapeto C, Vieira Barros DM. Nutrition and health as virtual class at Open University (Portugal): pedagogical strategies for higher education. International Journal of Educational Technology in Higher Education. 2019;16. 